\title{
PRIMITIVE GROUP RINGS
}

\author{
EDWARD FORMANEK ${ }^{1}$ AND ROBERT L. SNIDER
}

\begin{abstract}
Two theorems showing the existence of primitive group rings are proved.

THEOREM 1. Let $G$ be a countable locally finite group and $F$ a field of characteristic 0 , or characteristic $p$ if $G$ has no elements of order $p$. Then the group ring $F[G]$ is primitive if and only if $G$ has no finite normal subgroups.

THEOREM 2. Let $G$ be any group, and $F$ a field. Then there is a group $H$ containing $G$ such that $F[H]$ is a primitive ring.
\end{abstract}

All rings will be associative and have a unit. $R$ is a prime ring if $x R y \neq 0$ whenever $x$ and $y$ are nonzero elements of $R . R$ is a (left) primitive ring if there is a faithful irreducible (left) $R$-module. Every primitive ring is prime, but not conversely. A group is locally finite if every finitely generated subgroup is finite.

The prime group rings have been completely characterized by the following result:

THEOREM 1 (CONNELL $[1$, p. 675]). The group ring $R[G]$ is prime if and only if $R$ is a prime ring and $G$ has no finite normal subgroups.

Very little is known about primitivity in group rings. Almost no progress has been made toward answering the general question, "When is $R[G]$ primitive?" posed by Kaplansky [2] and Passman [3, p. 136]. Some negative statements are easy to make; for example, if $R$ is a field and $G \neq 1$ is abelian or finite then $R[G]$ is not primitive. Other negative results have been obtained by Alan Rosenberg [4]. But there were no examples of primitive $R[G]$ with $G \neq 1$. This paper proves two theorems in a positive direction.

THEOREM 2. Suppose $G$ is a countable locally finite group and $F$ is a field of characteristic 0 , or characteristic $p$ if $G$ has no elements of order $p$. Then $F[G]$ is primitive if and only if it is prime.

THEOREM 3. Suppose $G$ is a group and $F$ is a field. Then there is a group $H$ containing $G$ such that $F[H]$ is primitive.

Received by the editors February 29, 1972 and, in revised form, April 28, 1972. AMS 1969 subject classifications. Primary 1642, 1653, 2080.

Key words and phrases. Group ring, primitive ring, prime ring.

${ }^{1}$ The first author is a Canadian NRC postgraduate fellow supported by Grant A7171. 
Together with Connell's theorem, Theorem 2 provides a class of primitive group rings, while Theorem 3 says there are a lot of primitive group rings. The motivation for studying $F[G]$ for $G$ locally finite (and $F$ of acceptable characteristic) was provided by the following observation of Lance Small: $F[G]$ is von Neumann regular, so if $G$ has no finite normal subgroups, $F[G]$ is either

(1) a group ring which is primitive, or

(2) a prime von Neumann regular ring which is not primitive.

That is to say, $F[G]$ is something new! We are indebted to Susan Montgomery for communicating Small's observation.

THEOREM 4. The following are equivalent for any ring $R$.

(a) $R$ is left primitive.

(b) $R$ has a maximal left ideal which contains no nonzero two-sided ideals.

(c) $R$ has a proper left ideal $A$ such that $A+B=R$ for every nonzero twosided ideal $B$ of $R$.

The simple proof of Theorem 4 is left to the reader. Criterion (c) will be used in the proof of the next theorem.

A ring $R$ is Artinian semisimple if it is a finite direct product of complete matrix rings over division rings. Such an $R$ has a finite set $e_{1}, \cdots, e_{k}$ of irreducible central idempotents relative to which $R$ is an (internal) direct product of simple factors $R=R e_{1} \times \cdots \times R e_{k}$.

Theorem 2 is an immediate corollary of the following purely ring theoretic result, taking $R=F[G], R_{i}=F\left[G_{i}\right]$, where $G$ is expressed as the union of an ascending sequence of finite groups $G_{1} \subset G_{2} \subset G_{3} \cdots$.

THEOREM 5. Suppose $R$ is the union of an ascending sequence $R_{1} \subset R_{2} \subset R_{3} \cdots$ of Artinian semisimple rings. Then $R$ is primitive if and only if it is prime.

Proof. Since all primitive rings are prime, it is enough to show that if $R$ is prime, it is primitive.

Let $\left\{e_{1}, e_{2}, \cdots\right\}$ be an enumeration of all the irreducible central idempotents of all the $R_{i}$. It is worth noting that the $e_{i}$ commute with each other. Define a sequence of pairs $\left(R_{n_{1}}, f_{1}\right),\left(R_{n_{2}}, f_{2}\right), \cdots$, where $f_{i}$ is an irreducible central idempotent of $R_{n_{i}}$, inductively as follows.

Initial step. Let $f_{1}=e_{1}$ and $R_{n_{1}}$ be an $R_{i}$ in which $e_{1}$ is an irreducible central idempotent.

Inductive step. Assume $\left(R_{n_{1}}, f_{1}\right), \cdots,\left(R_{n_{k}}, f_{k}\right)$ have been chosen so that the following three conditions are satisfied:

(1) $e_{1} \in R_{n_{1}}, e_{2} \in R_{n_{2}}, \cdots, e_{k} \in R_{n_{k_{1}}}$.

(2) $e_{1} f_{1} \neq 0, e_{2} f_{2} \neq 0, \cdots, e_{k} f_{k} \neq 0$.

(3) $f_{1} \cdots f_{l} \neq 0$. 
Then choose $\left(R_{n_{k+1}}, f_{k+1}\right)$ as follows: Using the fact that $R$ is a prime ring choose $r \in R$ such that $e_{k+1} r f_{1} \cdots f_{k} \neq 0$, next, choose $n_{k+1}$ so large that $\epsilon_{k+1}, r \in R_{n_{k+1}}$; finally, let $f_{k+1}$ be an irreducible central idempotent of $R_{n_{k+1}}$ such that $\left(e_{k+1} r f_{1} \cdots f_{k}\right) f_{k+1} \neq 0$. Since $f_{k+1}$ commutes with $r f_{1} \cdots f_{k}$ it follows that $e_{k+1} f_{k+1} \neq 0, f_{1} \cdots f_{k+1} \neq 0$ and the three conditions are again satisfied for $k+1$.

Now let $A$ be the left ideal of $R$ generated by $\left\{1-f_{1}, 1-f_{2}, \cdots\right\}$.

(1) $A$ is a proper ideal of $R$.

If not, there is an integer $k$ and $r_{1}, \cdots, r_{k} \in R$ such that

$$
1=r_{1}\left(1-f_{1}\right)+\cdots+r_{k}\left(1-f_{k}\right) \text {. }
$$

$f=f_{1} \cdots f_{k} \neq 0$ and $\left(1-f_{i}\right) f=0$ for $i=1, \cdots, k$ since the $f_{i}$ are commuting idempotents. Multiplying $(*)$ on the right by $f$ yields the contradiction $f=1 \cdot f=0$.

(2) If $B$ is a nonzero two-sided ideal of $R, A+B=R$.

For $B$ must contain some $e_{i}$ and hence some $f_{i}$ since $e_{i} f_{i} \neq 0$ lies in the simple factor $R_{n_{i}} f_{i}$ of $R_{n_{i}}$.

$$
\therefore 1=\left(1-f_{i}\right)+f_{i} \in A+B .
$$

By Theorem $4, R$ is primitive.

We conclude with a proof of Theorem 3 . The construction we use yields an extravagantly large group $H$ containing the original group $G$. Passman has pointed out how to modify the construction so that $H$ and $G$ have the same cardinality in case $G$ is infinite. However, the given proof illustrates the idea in the most straightforward fashion.

Proof of Theorem 3. Define a sequence $\left\{G_{i}\right\}$ of groups and a sequence $\left\{M_{i}\right\}$ of modules inductively by

$$
\begin{array}{ll}
G_{1}=G, & M_{1}=F\left[G_{1}\right], \\
G_{2}=\operatorname{Aut}_{F}\left(M_{1}\right), & M_{2}=F\left[G_{2}\right] \oplus M_{1}, \\
G_{3}=\operatorname{Aut}_{F}\left(M_{2}\right), & M_{3}=F\left[G_{3}\right] \oplus M_{2},
\end{array}
$$

$G_{1} \subset G_{2} \subset G_{3} \cdots, M_{1} \subset M_{2} \subset M_{3} \cdots$, and we let $H=\bigcup G_{i}, M=\bigcup M_{i}$. Each $M_{i}$ is an $F\left[G_{i}\right]$-module via the obvious action and this action makes $M$ an $F[H]$-module. Moreover, $M$ is a faithful, irreducible $F[H]$-module, which shows that $F[H]$ is primitive. $M$ is faithful because each $M_{i}$ is a faithful $F\left[G_{i}\right]$-module. $M$ is irreducible since each $M_{i}$ is an irreducible $F\left[G_{i+1}\right]$-module. (In fact, $G_{i+1}$ acts transitively on the nonzero elements of $M_{i}$, so $H$ acts transitively on the nonzero elements of $M$.) 


\section{REFERENCES}

1. I. G. Connell, On the group ring, Canad. J. Math. 15 (1963), 650-685. MR 27 \#3666.

2. I. Kaplansky, "Problems in the theory of rings" revisited, Amer. Math. Monthly 77 (1970), 445-454. MR 41 \#3510.

3. D. S. Passman, Infinite group rings, Dekker, New York, 1971.

4. A. Rosenberg, On the primitivity of the group algebra, Canad. J. Math. 23 (1971), 536-540.

Department of Mathematics, Carleton University, Ottawa, Ontario, Canada

Department of Mathematics, University of Miami, Coral Gables, Florida 33124

Current address (R. L. Snider): Department of Mathematics, Northwestern University, Evanston, Illinois 60201 